\title{
Elementos Morfológicos Implicados en la Enseñanza de las Competencias para Lograr una Adecuada Venopunción Periférica
}

\author{
Morphological Elements Involved in the Teaching of Competences \\ to Achieve Adequate Peripheral Venipuncture
}

\author{
Edgar Giovanni Corzo Gómez; Olga Lucía Gómez Díaz²; Sergio Serrano Gómez \\ Mónica Saavedra Martínez ${ }^{3} \&$ Leidy Carolina Rojas Arenas ${ }^{4}$
}

CORZO, G. E. G.; GÓMEZ, D. O. L.; SERRANO, G. S.; SAAVEDRA, M. M. \& ROJAS, A. L. C. Elementos morfológicos implicados en la enseñanza de las competencias para lograr una adecuada venopunción periférica. Int. J. Morphol., 36(1):159-163, 2018.

RESUMEN: La venopunción periférica es un procedimiento muy común y necesario para la administración de múltiples sustancias. Cada enfermero(a) debe recibir la capacitación en varios aspectos que le permitan adquirir la competencia de realizar una venopunción segura. Uno de los elementos de mayor relevancia se relaciona con la anatomía aplicada, particularmente con la descripción de varios patrones de organización de las venas superficiales del antebrazo y su relación con estructuras neurovasculares cercanas. Los patrones de acuerdo a su distribución, podrían jugar a favor o en contra de las complicaciones durante o después de la punción. En este artículo describimos las respuestas de docentes de las escuelas de Enfermería de varias regiones de Colombia frente a la enseñanza de estos temas anatómicos. Sobresale el hecho que solamente en el 56,5\% de la escuelas de Enfermería se realiza la enseñanza completa de los conceptos anatómicos que servirán para mejorar la seguridad del paciente en el momento de una venopunción periférica.

PALABRAS CLAVE: Venopunción; Patrones venosos.

\section{INTRODUCCIÓN}

Las venopunciones periféricas son procedimientos que se llevan a cabo con mucha frecuencia en pacientes de todas las edades. Son útiles para administrar medicamentos, líquidos y sangre entre otros. Por lo tanto, el procedimiento de realizar una venopunción adecuada es un elemento de enseñanza de mucha importancia en los programas de Enfermería y en algunas especialidades médicas como anestesiología, urgencias y cuidado crítico.

El sitio más usado para las venopunciones corresponde a las venas superficiales del dorso de la mano y del antebrazo. Para la elección de la vena a canalizar se usan criterios como iniciar por el miembro no dominante, evitar zonas de flexión, priorizar venas distales sobre las proximales y elementos morfológicos generales como calibre, dirección, linealidad, longitud, entre otros (Arreguy-Sena \& Carvalho, 2008).
Sin embargo, existen algunos conceptos anatómicos que son más específicos y que requieren un mayor grado de conocimiento y entrenamiento por parte de la persona que realiza la venopunción. Tener en cuenta estos elementos repercute directamente en la seguridad del paciente y en la prevención de complicaciones durante o después de la punción (Lirk et al., 2004). Algunos de ellos han sido estudiados desde hace varias décadas y están relacionados con la cercanía de estructuras neurovasculares al sitio de punción (Vialle et al., 2001; Ramos, 2014) y otras descritas más recientemente se basan en la importancia clínica de los patrones que forman las venas superficiales del antebrazo y de la fosa cubital (del Sol et al., 2012; Yammine \& Eric, 2017). Estos patrones se han referenciado desde hace varios siglos, pero su estudio meticuloso se aumentó en el siglo veinte (del Sol \& Vásquez, 2009; Albustami et al., 2014; Corzo et al., 2010; Vucinic et al., 2016) (Fig. 1).

\footnotetext{
${ }^{1}$ Docente de Ciencias Básicas de la Universidad Autónoma de Bucaramanga, Colombia.

${ }^{2}$ Programa de Enfermería de la Universidad autónoma de Bucaramanga, Colombia.

${ }^{3}$ Docente de la Universidad Autónoma de Bucaramanga, Colombia.

${ }^{4}$ Estudiante de Enfermería de la Universidad Autónoma de Bucaramanga, Colombia.

Estudio financiado por la Universidad Autónoma de Bucaramanga dentro de su programa de convocatoria interna de investigaciones, con código: I56004
} 


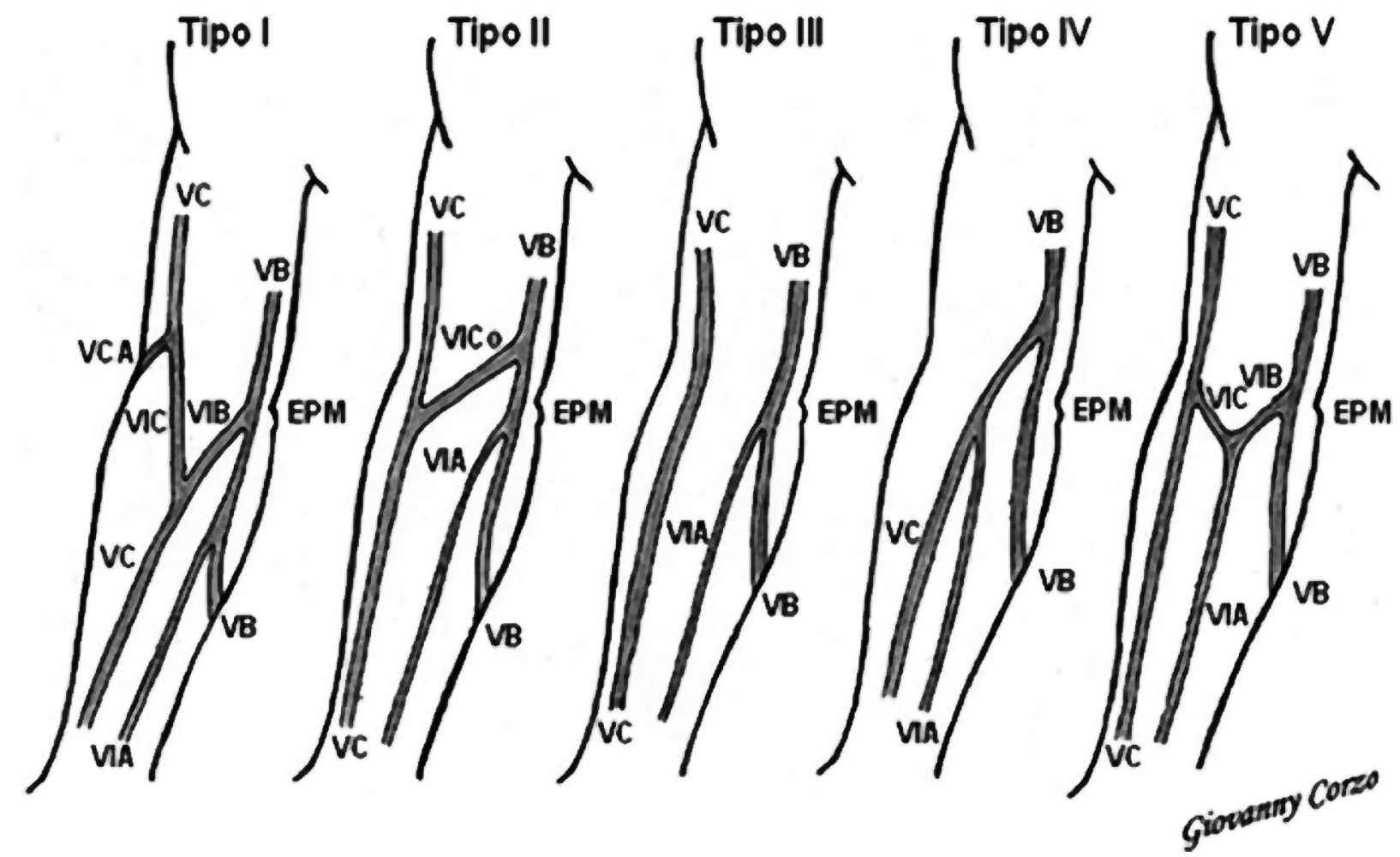

Fig. 1. Descripción de los patrones venosos de la fosa cubital según la propuesta de del Sol \& Vásquez (2009) y del Sol et al. (2012). VC: vena cefálica; VB: vena basílica; VCA: vena cefálica accesoria; VIC: vena mediana cefálica; VIB: vena mediana basílica; VIA: vena mediana del antebrazo; VICo: vena mediana del codo; EPM: epicóndilo medial.

Uno de los patrones venosos del antebrazo descrito con menos frecuencia es el denominado patrón IV, en el cual la vena cefálica es corta y finaliza en la vena basílica luego de hacer una curva pronunciada en el antebrazo (Kiray et al., 2013; Jain \& Yadav, 2015). Se han señalado posibles complicaciones luego de venopunciones de niños con presencia de este patrón (Corzo et al., 2016).

Para la valoración de los patrones venosos se han usado técnicas de compresión con torniquetes y recientemente se emplean transiluminadores con luz led que permite una visualización más clara (Lee et al., 2015; Corzo et al., 2016). Sin embargo, ocasionalmente en niños, aunque se aplique compresión, calor o luz led no es posible visualizar las venas superficiales, lo cual conlleva dificultades para el profesional que hace la punción aunada a dolor y riesgo de complicaciones en el niño.

En el trabajo expuesto a continuación consultamos a docentes de programas universitarios de Enfermería de la mayoría de las regiones geográficas de Colombia sobre la manera como enseñan los elementos anatómicos de las venas superficiales del antebrazo en pro de lograr las competencias para hacer una adecuada venopunción periférica.

\section{MATERIAL Y MÉTODO}

Se elaboró un formulario con el cual se consultó a docentes de programas de Enfermería de diferentes universidades de Colombia acerca de los elementos científicos y éticos que se enseñan a los estudiantes de Enfermería para alcanzar la competencia de realizar una adecuada venopunción periférica. Previa presentación del proyecto a las directoras de los programas, se aplicó el formulario vía internet, recibiendo respuestas de 71 docentes.

El formulario incluyó un módulo particular de aspectos anatómicos cuyos resultados se describen en el presente artículo. Las preguntas formuladas fueron:

1. ¿Se enseñan los diferentes patrones venosos superficiales que pueden encontrarse en el antebrazo?

2. ¿Se actualizan los nombres de las venas superficiales del miembro superior conforme los cambios de la terminología anatómica?

3. ¿Se enseña la relación de las ramas de los nervios ulnar, 
mediano, radial y musculo-cutáneo que se pueden lesionar durante una punción en la mano o el antebrazo?

4. ¿Se enseña la importancia de evitar puncionar las venas de la fosa cubital y metacarpianas, si se debe administrar tratamientos vesicantes? Debido a la dificultad que existe para identificar filtraciones en los sitios de flexuras.

Ante estas cuatro preguntas el docente podía escoger entre las siguientes respuestas: a) se enseña completamente; b) se enseña parcialmente; c) no se enseña.

La quinta y última pregunta señalaba lo siguiente:

5. Escriba el patrón anatómico que usted considera es más frecuente en la región donde es docente, usando los números romanos del I al V, teniendo en cuenta la imagen a continuación de un miembro superior derecho (Fig. 1):

Se realizó un análisis univariado descriptivo mediante medidas de frecuencia absoluta, frecuencia relativa (porcentaje) para cada una de las variables categóricas.

\section{RESULTADOS}

Las respuestas de los encuestados frente a las primeras cuatro preguntas se presentan en la Figura 2.

Pregunta 1. ¿Se enseñan los diferentes patrones venosos superficiales que pueden encontrarse en el antebrazo?

Pregunta 2. ¿Se actualizan los nombres de las venas superficiales del miembro superior conforme los cambios de la terminología anatómica?

Pregunta 3. ¿Se enseña la relación de las ramas de los nervios ulnar, mediano, radial y musculo-cutáneo que se pueden lesionar durante una punción en la mano o el antebrazo?

Pregunta 4. ¿Se enseña la importancia de evitar puncionar las venas de la fosa cubital y metacarpianas, si se debe administrar tratamientos vesicantes? Debido a la dificultad que existe para identificar filtraciones en los sitios de flexuras?

En la pregunta 5 el $34 \%$ de los encuestados respondió que el patrón venoso más frecuente era el I. El $31 \%$ respondió el patrón V. El 17 \% el patrón III. El 12,5 \% el patrón II y el 5,5\% el patrón IV.

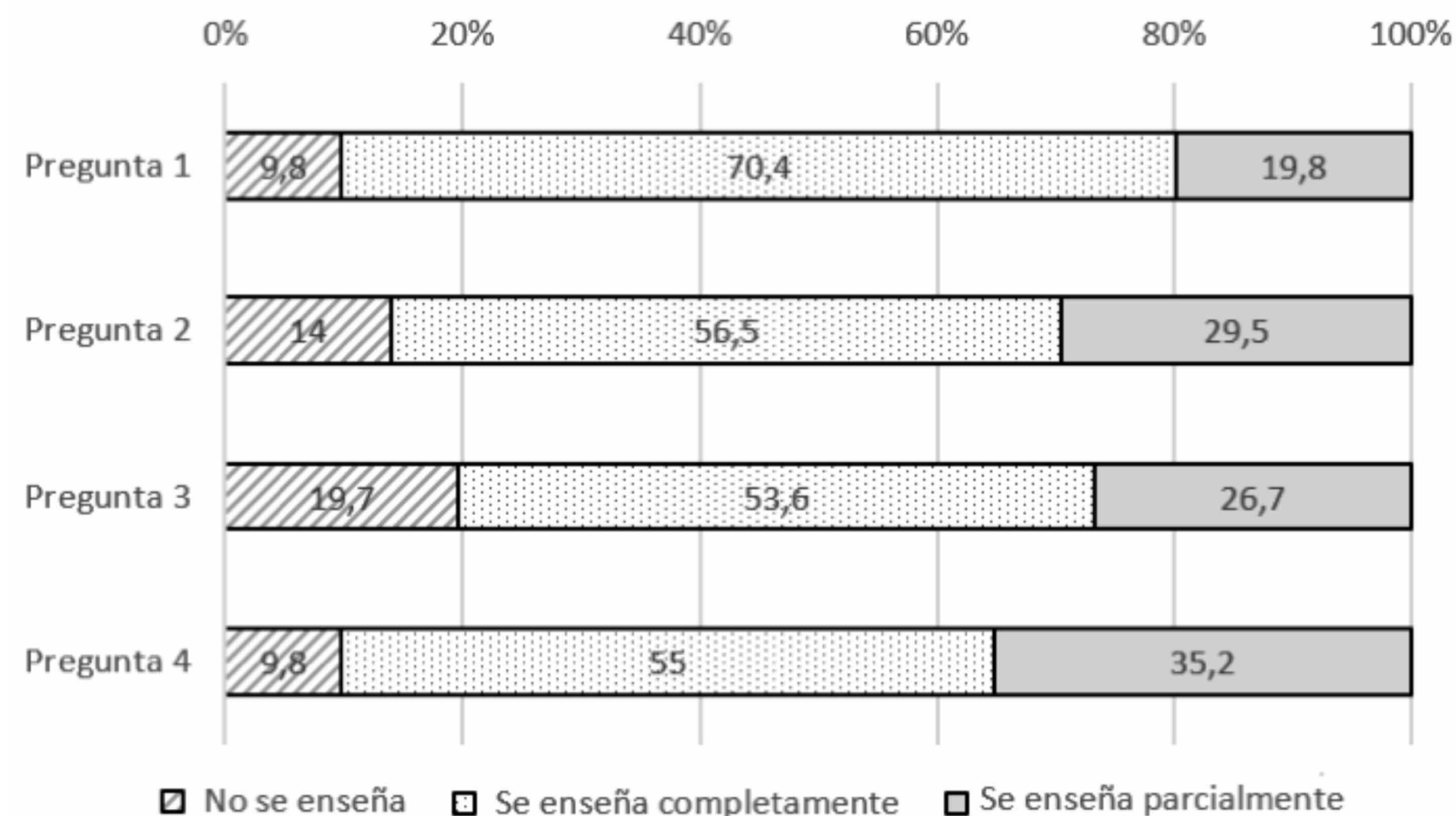

Fig. 2. Distribución de frecuencia de las respuestas de docentes de Enfermería frente a la enseñanza de elementos anatómicos implicados en la venopunción periférica. 


\section{DISCUSIÓN}

En relación a la primera pregunta: ¿Se enseñan los diferentes patrones venosos superficiales que pueden encontrarse en el antebrazo?. Resalta el hecho de que la respuesta "no se enseña" fue obtenida en el 9,8\% y la respuesta "se enseña parcialmente obtuvo un 19,8\% lo cual sugiere que, en la muestra estudiada, en cerca del $30 \%$ no se tiene en cuenta en la enseñanza las variaciones anatómicas del sistema venoso superficial del antebrazo y fosa cubital. Esto podría relacionarse con el hecho de que, a pesar de que el estudio de los patrones venosos se incrementó desde el siglo XX, en las actualizaciones de los textos clásicos de anatomía aún no se integran estos conceptos y se menciona la organización de las venas a partir de uno o dos patrones, en donde se le da una importancia aumentada al patrón $\mathrm{V}$ (Rouviére, 1924; Netter, 2006; Moore \& Dalley, 2009). Lo anterior fue motivado por el hecho de que durante mucho tiempo se le asignó una gran frecuencia de presentación al patrón en "M" (V) como fruto de un error conceptual que confundió el patrón $\mathrm{V}$ con el I. Esto ha sido señalado por autores como Salvi (1932) y del Sol \& Vásquez (2009). Por lo anterior es muy importante concientizar a los directores de programas de Enfermería y docentes en la información actualizada existente ahora sobre los patrones venosos del antebrazo y sus implicaciones clínicas.

En la pregunta dos: ¿Se actualizan los nombres de las venas superficiales del miembro superior conforme los cambios de la terminología anatómica?. Se evidencia que la respuesta "se enseña completamente" fue respondida por el $56,5 \%$ de los docentes, es decir que cerca de la mitad de los estudiantes no reciben una actualización sobre los cambios en la terminología anatómica. Este aspecto señala un problema mencionado comúnmente por docentes de medicina y enfermería, en razón a que no hay claridad total en los nombres de las venas ya que han ido cambiando a través de las décadas generando confusiones. Incluso se han generado discrepancias importantes con las ediciones de Nómina Anatómica y Terminologia Anatomica de acuerdo al Federative International Programme on Anatomical Terminology, FIPAT (del Sol \& Vásquez, 2014). Desafortunadamente las agrupaciones encargadas de asignar los nombres a las estructuras anatómicas son varias, y no siempre están de acuerdo en la denominación. A esto se le suma que las actualizaciones de las ediciones de los textos anatómicos son muy lentas.

En la pregunta tres: ¿Se enseña la relación de las ramas de los nervios ulnar, mediano, radial y musculo-cutáneo que se pueden lesionar durante una punción en la mano o el antebrazo?. La suma de las respuestas "no se enseña" y "se enseña parcialmente" superan el $50 \%$. Este aspecto es muy importante dado que el desconocer la cercanía anatómica de estas estructuras nerviosas a las venas superficiales puede generar lesiones y eventos iatrogénicos en el momento de la venopuncion. Lo anterior, se vuelve más delicado en los bebes, ancianos y pacientes con alteración de la conciencia que no pueden expresar el dolor o la incapacidad que produce la punción de un nervio. Esto ha sido señalado por varios autores (Vialle et al.; Ramos). Aunque es claro que en las asignaturas de anatomía se explican las ramas del plexo braquial y sus relaciones topográficas, sería adecuado que en los niveles en los que se enseñan las competencias para la venopunción, se retomaran estos conceptos anatómicos direccionados a aumentar la seguridad de los procedimientos.

Lo mencionado en la pregunta cuatro: ¿Se enseña la importancia de evitar puncionar las venas de la fosa cubital y metacarpianas, si se debe administrar tratamientos vesicantes debido a la dificultad que existe para identificar filtraciones en los sitios de flexuras?. ¿Es algo que se enfatiza en la mayoría de las guías de venopunción debido a que las agujas ubicadas en sitios de flexión producen flebitis, obstrucción e infiltraciones, en particular si se aplican sustancias lesivas al endotelio?. En nuestros resultados se enseña completamente sólo en un $55 \%$, cifra que consideramos baja, teniendo en cuenta riesgo que implica para el paciente.

En la pregunta cinco: "señale el patrón anatómico que usted considera es más frecuente en la región donde es docente, usando los números romanos del I al V" (teniendo en cuenta la Fig. 1), el $34 \%$ de los encuestados respondió que el patrón venoso más frecuente era el I. El 31 \% respondió el patrón V. El 17 \% el patrón III. El 12,5 \% el patrón II y el 5,5 \% el patrón IV. Los amplios estudios realizados desde el siglo pasado han mostrado que los patrones venosos de acuerdo al orden de frecuencia son así: Predominan los patrones I y II seguido de los patrones III y IV y con menor frecuencia el patrón V (del Sol \& Vásquez, 2009; Albustami et al.; Vucinic et al.). Según Corzo et al. (2010, 2016) en población colombiana el patrón I oscila entre el $14 \%$ y el $30 \%$; el patrón II entre $19 \%$ y el $24 \%$; el patrón III entre el $26 \%$ y el $37 \%$; el patrón IV entre el $10 \%$ y el $12 \%$ y el patrón $\mathrm{V}$ entre el $4 \%$ y el $8 \%$. Cabe resaltar que la alta frecuencia del patrón III se relacionó más con las mujeres.

En conclusión, observamos que, de las primeras cuatro preguntas, en tres de ellas, la enseñanza completa de los conceptos anatómicos que sirvan para mejorar la seguridad del paciente en el momento de una venopunción se encuentra entre $53,6 \%$ y el $56,5 \%$, sólo en la primera pregunta la enseñanza completa llega al 70,4 \%. Lo cual sugiere que la enseñanza para los estudiantes de enfermería, de conceptos 
anatómicos claves para garantizar la seguridad de los pacientes durante una venopunción periférica, se ve comprometida en un porcentaje importante. Este hecho podría tener varias causas, por ejemplo, la falta de actualización de los docentes de anatomía y de los docentes implicados directamente en la enseñanza de la venopunción, la falta de actualización de los textos de anatomía y la confusión actual sobre la denominación de las venas desde la terminología anatómica.

\section{AGRADECIMIENTOS}

Estudio financiado por la Universidad Autónoma de Bucaramanga dentro de su programa de convocatoria interna de investigaciones con código: I56004

A las Facultades de Enfermería de las universidades de Colombia cuyos docentes amablemente participaron con el diligenciamiento de la encuesta.

CORZO, G. E. G.; GÓMEZ, D. O. L.; SERRANO, G. S.; SAAVEDRA, M. M. \& ROJAS, A. L. C. Morphological elements involved in the teaching of competences to achieve adequate peripheral venipuncture. Int. J. Morphol., 36(1):159-163, 2017.

SUMMARY: Peripheral venipuncture is a very common and necessary procedure for the administration of multiple substances. Each Nurse must receive training in several aspects that allows him/her to acquire the competence to perform a safe venipuncture. One of the most relevant elements is related to applied anatomy, particularly with the description of various organization patterns of the superficial veins of the forearm, and their relation to nearby neurovascular structures. These patterns according to their distribution, could play for, or against complications during or after puncture. In this article, we describe the responses of teachers from the Nursing Schools of several regions of Colombia while teaching these anatomical subjects. It is noteworthy, that only $56,5 \%$ of Nursing Schools included complete instruction of anatomical concepts that improve patient safety at the time of a peripheral venipuncture. Therefore, nearly $50 \%$ of students do not receive information on updated anatomical terminology.

KEY WORDS: Venopuncture; Venous patterns.

\section{REFERENCIAS BIBLIOGRÁFICAS}

AlBustami, F.; Altarawneh, I. \& Rababah, E. Pattern of superficial venous arrangement in the cubital fossa of adult Jordanians. Jordan Med. J., 48(4):269-74, 2014.

Arreguy-Sena, C. \& Carvalho, E. C. Classificação de veias superficiais periféricas de adolescentes, adultos e idosos pela técnica Delphi. Rev. Latinoam. Enferm., 16(1):86-94, 2008.
Corzo, G. E. G.; Gómez, D. O. L.; Niño, M. M. E.; Rey, T. R. J. \& Pedraza, D. L. J. Distribution pattern of the veins of the cubital fossa in a sample of people born in Bucaramanga, Colombia. Int. J. Morphol., 28(4):1011$8,2010$.

Corzo, G. E. G.; Robles, C. M. I.; Díaz, D. A. M. \& Osma, Z. S. E. Relationship between surface forearm vein pattern and complications of peripheral and central venous catheterization in a sample of children treated in the Pediatric Intensive Care Unit of Santander Colombia. Int. J. Morphol., 34(3):885-9, 2016.

del Sol, M. \& Vásquez, B. Median cephalic and median basilic veins. Why their exclusion from Terminologia Anatomica? Int. J. Morphol., 32(2):721-4, 2014.

del Sol, M. \& Vásquez, B. Superficial veins of the cubital fossa. Anatomical, clinical and anthropological aspects. Int. J. Morphol., 27(2):527-38, 2009.

del Sol, M.; Lillo, E.; Lobos, L. \& Vásquez, B. Study of the veins of the cubital fossa by helical computed tomography and its clinical application. Int. J. Morphol., 30(1):64-9, 2012.

Jain, T. \& Yadav, S. K. Case study: Variation of superficial veins pattern of upper limb found in dissection. Int. Ayurvedic Med. J., 3(7):2223-5, 2015.

Kiray, A.; Ergür, I.; Tayefi, H.; Bagriyanik, H. A. \& Bacakoglu, A. K. Anatomical evaluation of the superficial veins of the upper extremity as graft donor source in microvascular reconstructions: a cadaveric study. Acta Orthop. Traumatol. Turc., 47(6):405-10, 2013.

Lee, H.; Lee, S. H.; Kim, S. J.; Choi, W. I.; Lee, J. H. \& Choi, I. J. Variations of the cubital superficial vein investigated by using the intravenous illuminator. Anat. Cell Biol., 48(1):62-5, 2015.

Lirk, P.; Keller, C.; Colvin, J.; Colvin, H.; Rieder, J.; Maurer, H. \& Moriggl, B. Unintentional arterial puncture during cephalic vein cannulation: case report and anatomical study. Br. J. Anaesth., 92(5):740-2, 2004.

Moore, K. L. \& Dalley, A. F. Anatomía con Orientación Clínica. Buenos Aires, Médica Panamericana, 2009.

Netter, F. H. Atlas de Anatomia Humana. $4^{\mathrm{a}}$ ed. Philadelphia, Saunders/ Elsevier, 2006.

Ramos, J. A. Lesión del nervio cutáneo antebraquial lateral relacionada con la venopunción: ¿qué debemos saber? Rev. Bras. Anestesiol., 64(2):131-3, 2014.

Rouviére, H. Anatomie Humaine. Vol. 2. Paris, Masson, 1924.

Salvi, G. Angiología. En: Balli, R. \& Bertelli, D. Trattato di Anatomía Umana. Vol. 3. $2^{\text {a }}$ ed. Milano, Casa Editrice Dottor Francesco Vallardi, 1932.

Vialle, R.; Pietin-Vialle, C.; Cronier, P.; Brillu, C.; Villapadierna, F. \& Mercier, P. Anatomic relations between the cephalic vein and the sensory branches of the radial nerve: How can nerve lesions during vein puncture be prevented? Anesth. Analg., 93(4):1058-61, 2001.

Vucinic, N.; Eric, M. \& Macanovic, M. Patterns of superficial veins of the middle upper extremity in Caucasian population. J. Vasc. Access, 17(1):87-92, 2016

Yammine, K. \& Eric, M. Patterns of the superficial veins of the cubital fossa: A meta-analysis. Phlebology, 32(6):403-14, 2017.

Dirección de correspondencia:

Edgar Giovanni Corzo Gómez

Universidad Autónoma de Bucaramanga

Calle 157 número 19-55 Cañaveral Parque

Floridablanca

Santander

COLOMBIA

Recibido : 09-07-2017

Aceptado: 11-10-2017

Email: ecorzo@unab.edu.co 